\title{
Parity of publication for psychiatry
}

Sayinthen Vivekanantham, Rebecca Strawbridge, Riaz Rampuri, Thivvia Ragunathan and Allan H. Young

\section{Background}

There is an established disparity between physical and mental healthcare. Parity of research outputs has not been assessed internationally across influential medical journals.

\author{
Aims \\ To assess parity of publication between physical and mental \\ health, and within psychiatry.
}

\section{Method}

Four major medical disciplines were identified and their relative burden estimated. All publications from the highestimpact general medical journals in 2001, 2006 and 2011 were categorised accordingly. The frequency of psychiatry, cardiology, oncology and respiratory medicine articles were compared with the expected proportion (given illness burdens). Six subspecialties within psychiatry were also compared.

\section{Results}

Psychiatry was consistently and substantially underrepresented; other specialties were overrepresented. Dementia and psychosis demonstrated overrepresentation, with addiction and anxiety disorders represented proportionately and other disorders underrepresented. The underrepresentation of mood disorders increased more recently.

\section{Conclusions}

There appears to be an important element of disparity of esteem; further action is required to achieve equivalence between mental and physical health research publications.

\section{Declaration of interest}

None.

\section{Copyright and usage}

(c) The Royal College of Psychiatrists 2016.
There is a long and well-documented history of stigma in mental health that has now resulted in the objective of achieving 'parity of esteem' between mental and physical health. ${ }^{1}$ Millard \& Wessely depicted the current situation and need for parity, alongside the challenges and considerations necessary to attaining equivalent healthcare. ${ }^{2}$ The interactions between physical and mental health and the burdens of illness must be accounted for in assessments of parity. However, it is also important that parity is measured and achieved at the research level, from which treatments ultimately arise: if research is inadequately resourced, then progress with improving management of illnesses is hindered and this heavily influences quality of care. We propose that the term 'parity of publication' be used to describe the comparison of reputable or influential research communication between physical and mental health, and this requires examination and monitoring in the pursuit of parity of esteem. It is unlikely that all areas within mental health are equivalently represented in the influential research literature proportional to illness burdens; thus, it is additionally valuable to assess parity of publication between subcategories of psychiatry (for example, affective disorders compared with psychotic disorders). We investigated the state of equivalence between psychiatry and other medical specialties in publication rates over a 10 -year period across research journals with the highest impact factors, predicting that mental health would be underrepresented compared with physical health areas when accounting for burden of disease. Our second hypothesis explored the relative representation of different categories within mental health, also standardising publication rates with burden of disease.

\section{Method}

We used Martin Prince's seminal 2007 article as a basis for the calculations and definitions of medical specialties and disease burden. ${ }^{3}$ The medical specialties selected for comparison with psychiatry were cardiology, oncology and respiratory medicine. These represent the categories imposing the greatest health burdens (excluding sense organ impairments which were considered to be too broad). We sought to identify the research journals with greatest influence on healthcare provision; thus we selected the top ten journals in terms of impact factor in the broad category of general and internal medicine (most relevant to the medical specialties to be compared) as documented on Journal Citation Reports outlined on the online Web of Knowledge Journal Index (http://wok.mimas.ac.uk) in June 2012, when the search was conducted. Within these journals articles were studied for three separate years of publication $(2001,2006,2011)$, to depict the pattern over a decade. The total numbers of papers in each year relating to psychiatry, cardiology, oncology and respiratory medicine were categorised according to medical specialty by reading titles and abstracts through the respective online journal archives. Articles were categorised in multiple disease areas if the focus crossed categories (for example, investigating depression in cardiovascular disease). The type of paper (original research, correspondence, review article, editorial or other publication type) was recorded, alongside the total number of articles published for each journal in each year. The 'other publication' category included case records, corrections, media reviews, essays, news and additional unusual types of article.

Burden estimates for each specialty were taken from a World Health Organization (WHO) national survey in 2005, as reported by Prince et al. ${ }^{3}$ The contribution of neurological disorders and epilepsy was excluded from the analysis. Psychiatry articles identified were subcategorised into the following groups: addiction, anxiety disorders, dementia, mood disorders, psychotic disorders and other disorders. Papers that made reference to more than one subspecialty were included in the 'other disorders' group. Expected representation of subspecialties was calculated from the WHO Global Disease Adjusted Life Years (DALYs) estimates in $2011^{4}$ 


\section{Statistical analysis}

Expected percentages of each specialty were calculated by multiplying each specialty's burden of disease proportion (gathered using the methods outlined above) by the total frequency of articles published across the four specialties (and six subspecialties within psychiatry) for each year studied. The expected values for each group at each time point were compared with the observed values (actual frequency of publication for each comparison) using goodness of fit chi-squared tests. Where results were significant $(P<0.05)$ planned post hoc comparisons were conducted, examining standardised residuals for each group within each analysis to appraise which categories contributed to the significant observation. These analyses provided us with an assessment of the relative publication frequencies between groups. Primary analyses were conducted using IBM SPSS statistical software (version 21.0, MS Windows). As a secondary aim we examined the distribution of different types of articles (i.e. editorial, perspective, comments, correspondence, original articles, reviews, letters and other publications).

\section{Results}

In total 32642 articles were published in the ten journals included in analyses across 2001, 2006 and 2011, of which 575 (1.76\%) concerned psychiatry. Of the total, 4121 articles were allocated to the four categories examined, leaving 28521 medical articles uncategorised (Table 1). There was considerable variation within the journals in the representation of psychiatry: the Cochrane Database of Systematic Reviews was ranked lowest by impact factor and had the highest proportion of psychiatry publications at $5.94 \%$, whereas the BMJ (ranked sixth by impact factor) had the lowest percentage of psychiatry publications at $1.11 \%$. The relative burden of disease figures were $26 \%, 20 \%, 11 \%$ and $8 \%$ for psychiatry, cardiology, oncology and respiratory medicine respectively. Given the total publication rates of these four categories, expected values for each discipline were in the range 5.3-7.3\% (psychiatry), 4.1-5.6\% (cardiology), 2.2-3.1\% (oncology) and $1.6-2.3 \%$ (respiratory medicine) over the three years.

Psychiatry was underrepresented in publication rates (compared with expected rates based on burden) in contrast to cardiology, oncology and respiratory medicine which all demonstrated more actual than expected publications. This was demonstrated in $2001\left(\chi^{2}=57.94\right.$, d.f. $\left.=3, P<0.0001\right), 2006$ $\left(\chi^{2}=51.05\right.$, d.f. $\left.=3, P<0.0001\right)$ and $2011\left(\chi^{2}=35.68\right.$, d.f. $=3$, $P<0.0001)$; Fig. 1. Post hoc standardised residual values demonstrated that psychiatry's underrepresentation and the overrepresentation of oncology and respiratory medicine all contributed substantially to the variation in all years; the only area that did not contribute to the variance was cardiology for all years studied. In 2001 the proportion of psychiatric publications was $33 \%$ of the expected number. This figure increased to $43 \%$ in 2006 and in 2011 it decreased to $38 \%$. In contrast, the representation of cardiology in 2001 was $122 \%$ of the expected proportion, in 2006 it was $104 \%$ and in 2011 it was $127 \%$. Oncology was at $132 \%$ in 2001 and $164 \%$ of the expected frequency in both 2006 and 2011, whereas respiratory medicine was double $(200 \%)$ in 2001, $178 \%$ in 2006 and $156 \%$ in 2011.

Within psychiatry publications, most areas in 2001 showed less actual than expected rates of publication (addiction, anxiety, mood disorders and other disorders), although both dementia and psychotic disorders showed the opposite outcome. Overall, variance between subspecialties was highly significant $\left(\chi^{2}=249.31\right.$, d.f. $\left.=5, P<0.0001\right)$. The pattern was similar in $2006\left(\chi^{2}=173.62\right.$, d.f. $\left.=3, P<0.0001\right)$ and $2011\left(\chi^{2}=86.15\right.$, d.f. $=3, P<0.0001)$. The standardised residuals calculated for these comparisons also identified relative stability of results: in 2001 dementia and psychotic disorders contributed significantly (overrepresentation) whereas underrepresentation of the 'other disorder' category reached a significant residual value, and this pattern remained in 2006. In 2011 dementia and psychotic disorders showed similarly significant contributions to the variance, whereas the category comprising other disorders was not a significant contributor. In contrast, mood disorders were markedly underrepresented.

As well as constituting the highest burden in neuropsychiatry, mood disorders was the subspecialty with the most publications (excluding other disorders) in both 2001 (24\%) and 2006 (26\%); Fig. 2. Dementia contributed the most publications in $2011(20 \%)$ which was 383\% higher than its expected output, whereas the observed representation of depression was only $56 \%$ of the expected value. The overrepresentation of dementia was consistent over the 10-year period, and this was also detected for psychotic disorders whose overrepresentation ranged from $234 \%$ of the expected proportion in 2011 to $462 \%$ in 2001 .

\section{Secondary findings}

The majority of psychiatry papers in 2001 were in the 'other disorder' category, comprising $44.7 \%$ of its articles that year, whereas in 2006 just $12.0 \%$ of psychiatry papers were categorised as other publication types. A greater percentage of papers in 2006 were reviews (39.5\%). In 2011 both of these types were well represented: reviews at $28.9 \%$ and other publication type at

Table 1 Details of journals analysed (summed data from 2001, 2006 and 2011)

\begin{tabular}{|c|c|c|c|c|c|c|}
\hline \multirow[b]{2}{*}{ Top ten journals } & \multirow[b]{2}{*}{ Impact factor } & \multicolumn{5}{|c|}{ Number of articles } \\
\hline & & Total & Psychiatry & Cardiology & Oncology & $\begin{array}{l}\text { Respiratory } \\
\text { medicine }\end{array}$ \\
\hline New England Journal of Medicine & 53.486 & 3379 & 71 & 273 & 281 & 171 \\
\hline Lancet & 33.633 & 6643 & 97 & 349 & 300 & 232 \\
\hline JAMA & 30.011 & 4717 & 81 & 168 & 170 & 132 \\
\hline Annals of Internal Medicine & 16.729 & 1800 & 28 & 145 & 47 & 65 \\
\hline PLOS Medicine & 15.617 & 615 & 16 & 12 & 5 & 31 \\
\hline BMJ & 13.471 & 8385 & 93 & 157 & 165 & 109 \\
\hline Annual Review of Medicine & 12.457 & 97 & 5 & 10 & 14 & 4 \\
\hline Archives of Internal Medicine & 10.639 & 1819 & 31 & 201 & 52 & 64 \\
\hline Canadian Medical Association Journal & 9.015 & 3353 & 44 & 78 & 58 & 39 \\
\hline Cochrane Database of Systematic Reviews & 6.186 & 1834 & 109 & 84 & 28 & 102 \\
\hline
\end{tabular}



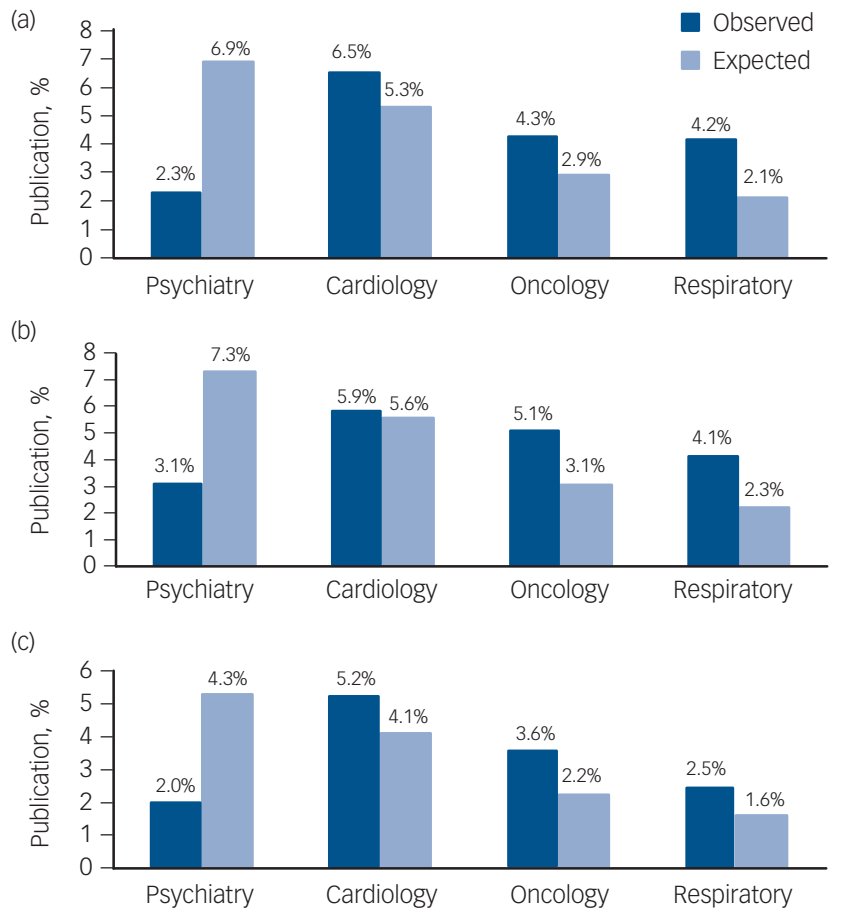

Fig. 1 Parity of publication in psychiatry, cardiology, oncology and respiratory medicine: (a) in 2001; (b) in 2006; (c) in 2011.

$34.9 \%$. However, in each year original research was consistently underrepresented compared with other disciplines. Cardiology showed a particularly high percentage of original articles in all years examined. Although oncology had the highest proportion of correspondence publications in all years, overall percentages across the different types of article were fairly evenly distributed. Respiratory illnesses also showed stable distribution across article types, with consistently high proportions of both original articles and other articles represented; this was most pronounced in 2011 when these two categories each comprised $24.9 \%$ - almost half of the relevant papers. Initially, psychiatry was well represented in terms of editorial papers; this reduced in more recent years (from $6.8 \%$ to $4.7 \%$ and $5.9 \%$ ) and showed an opposing pattern to oncology editorials (from $4.5 \%$ to $6.8 \%$ and $11.6 \%$ ).

\section{Discussion}

This is the first study to demonstrate a consistent underrepresentation of mental health compared with three major areas of physical health in the publication of international, high-quality research in general medical journals over a decade. Psychiatry exhibited the greatest burden of disease, and consistently the fewest articles published of the four medical disciplines. This disparity may be most pronounced for original research articles, which perhaps highlights the problem further. Whereas the other three disciplines were overrepresented based on the burden estimate, cardiology was reliably the closest to parity. Within mental health, results showed fairly stable trends across the 10 -year period, with dementia and psychotic disorders overrepresented and those in the 'other disorders' category underreported. There are indications that mood disorders (which represent the most burdensome category within mental health) have become less well represented in recent years. Publication rates

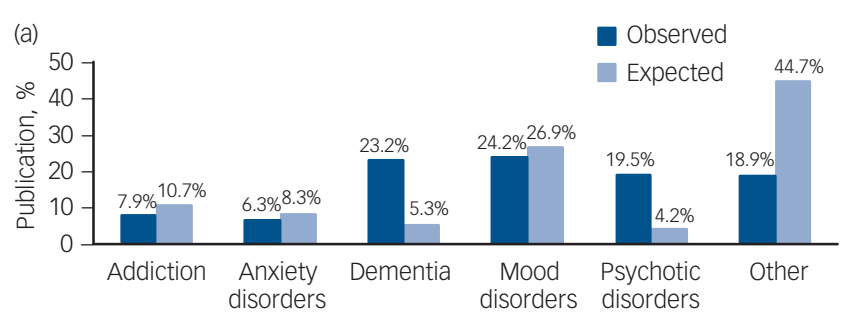

(b)

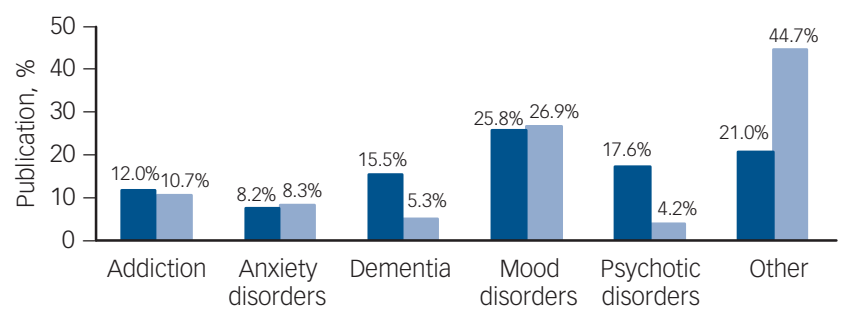

(c)

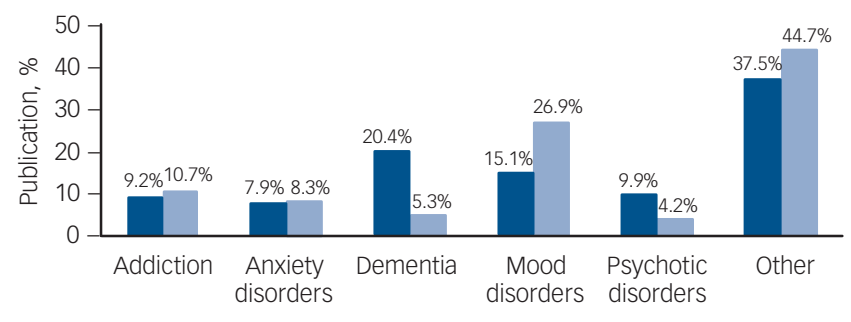

Fig. 2 Parity of publication in addiction, anxiety, dementia, mood disorders, psychosis and other disorders: (a) in 2001; (b) in 2006; (c) in 2011.

of mental health-focused research varied between journals, and this may reflect our choice of publication sources: although this is the general medical category, some of these are journals specifically communicating internal medicine (of which mental health is not the focus). Despite this, the internal medicine journals we investigated did not demonstrate lower proportions of psychiatry articles than the other, more general journals.

Previous reports have demonstrated that low- and middleincome countries produce substantially less psychiatric research than Europe and America, ${ }^{5}$ thus the research published may also demonstrate ethnocentricity. Less funding for psychiatry than for other disciplines has been reported, ${ }^{6}$ and this is probably a contributory factor to the reduced research output that we have identified. Low numbers of psychiatric researchers may be partly a consequence of funding scarcity, or of social stigma (which appears to contribute to an overall unpopular perspective of psychiatry within the medical profession). ${ }^{7,8}$ Another putative contributor to low parity of publication is a reduced willingness of high-impact journals to publish psychiatric articles, perhaps derived from stigma-related factors. Psychiatric research might be further complicated by its nature: an absence of clear biological markers of psychiatric disorders has led to the use of a criteriabased diagnostic system, which gives rise to challenges including the comparability of symptom ratings and diagnoses. This often leads to increased heterogeneity in research findings and reduced statistical significance of results, and ultimately can succumb to publication bias. ${ }^{9}$ The inability to make consistently accurate diagnoses in psychiatry could also perpetuate stigma across medical and academic professions. ${ }^{10}$ Stigma within mental health itself may impede researcher willingness to submit papers to high-impact general medical journals (if mental health is 
perceived to be too niche an interest), and there may be a higher frequency of publication opportunities in psychiatry-specific journals than is the case in other areas of medicine. Methodological challenges encountered with much psychiatric research can result in underpowered studies which are less likely to be accepted for publication in high-impact research journals. It is possible that (whatever the causes for low parity of publication), the underrepresentation of psychiatric research may self-perpetuate owing to ongoing maintaining factors.

Within psychiatry, the category of 'other disorders' was the most underrepresented. This group includes personality disorders, eating disorders and developmental disorders, as well as a large number of conditions that are generally less well understood and/or prevalent, which we were not able to analyse. It must be noted that articles pertaining to more than one psychiatry category were also categorised in the 'other disorders' group, which only serves to magnify our finding, and it is essential that the specific diagnoses within this group are addressed in future investigations. Compared with other areas of psychiatry, anxiety disorders do not appear to require increased prioritisation, as has been suggested by Christensen et al. ${ }^{11}$ Their article also documented the recently increased funding in Australia in areas such as psychosis and dementia, both of which we found to be overrepresented. Psychotic disorders have notoriously been neglected in research in the past, and dementia has well-documented projections of burden increases in the future, which may explain the above phenomena. Future methods for burden calculations of disease may yield greater parity within psychiatry if accounting for projected change in illness burden (although the measurement of this presents a challenge). Alternatively, the marked frequency of dementia articles compared with other areas within psychiatry may denote a different kind of burden, as dementia has a high contribution to mortality in terms of years of life lost, rather than DALYs. ${ }^{3}$ This could reflect the priority given to early mortality rather than suffering with a debilitating condition, which Prince et al speculates is typically prioritised in medicine. We note that our use of DALYs to approximate burden might have been altered if mortality had been prioritised. However, the Global Burden of Disease Study in 2010 implied that the values reported by Prince et al in 2007 might be an underestimation of the true burden of mental illness. ${ }^{12}$

\section{Study limitations}

We note limitations of this study in terms of the time-points and scope of influential publications that we were not able to measure. The selection of journals may not truly represent those that influence healthcare provision, and impact factors have previously been speculated to be trivial. ${ }^{13}$ Further research might investigate publication rates based on the most cited articles. It is important to highlight that only a small proportion of the total papers published in the ten journals were considered in this article (approximately 13\%). It is also conceivable that there are specific psychiatry journals that confront the research gaps identified in this study, and the intrinsic value that such journals have in driving forward the discipline must be appreciated. Owing to the extremely high levels of statistical significance in this paper, and the quantity of supporting evidence, we argue that disparity of publication in psychiatry is likely to be a valid finding.

\section{Study implications}

The clear discrepancy between physical and mental health treatment has triggered numerous initiatives in recent years, aimed at reducing discrimination and stigma towards psychiatry.
In 2001 WHO dedicated its World Health Report to mental health, illustrating the necessity of research in enabling global improvements in mental health resource utilisation. ${ }^{14}$ There has been a substantial movement to increase publication of mental health research in The Lancet, with a series of papers from the internationally diverse Lancet Mental Health Group. ${ }^{15}$ It has also been suggested that measuring stigma in research studies will help reduce disparities in equality. ${ }^{16}$ More generally in mental health, clinical improvement programmes have been piloted, ${ }^{17}$ and there have been increased efforts to tackle stigma: an evaluation of the UK Time to Change programme indicated that public intended behaviours and attitudes - but not knowledge about mental health - improved between 2009 and 2012. ${ }^{18}$ Despite these efforts, it appears that parity of publication has not progressed positively between 2001 and 2011. Research is a tool for change, forming the basis of mental health policies and clinical practice, and it also acts as an indirect measure for the relative priority given to different conditions. Our results highlight the need to break the barriers that impede publication, and concrete steps could be taken toward the goal of achieving parity of publication, which we feel could facilitate the overall prioritisation of mental health in medicine. Lobbying research bodies to allocate appropriate proportions of funding to psychiatry is a solution, and although there is currently a worldwide shortage of human resources for mental health, strategies have been suggested. ${ }^{19}$ Running antistigma sessions for medical students is one possibility that has been assessed preliminarily. ${ }^{20}$

Although some evidence suggests a publication bias from journal editorial boards associated with gender, ethnicity or towards research that aligns with editors' own interests, ${ }^{21}$ we could not identify any investigation measuring representation of different medical specialties and its relationship to discipline representation of articles published. We believe that assessing this is important; if a disparity of esteem at the editorial level is present, this may be having widespread implications (if psychiatry is not highly regarded by the editor of an influential journal, this could affect a population of medical professionals), then identifying the problem could facilitate the use of a recruitment drive for underrepresented medical specialties within general healthcare journals, which might improve parity of publication. We found that editorial articles were less prevalent in more recent years, which may be a sign of current disparities at this level. Research journals remain integral to the advancement of specialties; ${ }^{22}$ indeed, central to evidence-based medicine is the strength of high-quality research to inform clinical practice, ${ }^{23}$ and without articles reaching wider audiences we suggest that this impedes progression towards parity of esteem.

\section{Future directions}

We recommend that to achieve parity of publication (and ultimately parity of esteem overall between mental and physical health) a multi-pronged approached is required. Strategies to increase the proportion of research and treatment funding in psychiatry, and to reduce bidirectional biases between researchers, editors and publishers, can function alongside recruitment drives in medicine to psychiatry and to research careers, in addition to continuation of international efforts to reduce discrimination in mental health. The implications for parity of esteem are undoubtedly vast. We know that disparities are found at many levels within medicine and across society; from a lack of treatment seeking and provision, ${ }^{24}$ to healthcare funding. ${ }^{19}$ We postulate that pursuit of equivalent funding and research representation of mental health in academia is a means to prioritise mental health, and consequently public health and well-being. 
Sayinthen Vivekanantham, BSc, Imperial College School of Medicine, London; Rebecca Strawbridge, MSc, Centre for Affective Disorders, Institute of Psychiatry, Psychology and Neuroscience, London; Riaz Rampuri, BSc, Thivvia Ragunathan BSC, Imperial College School of Medicine, London; Allan H. Young, FRCPsych, Centre for Affective Disorders, Institute of Psychiatry, Psychology and Neuroscience, London, UK

Correspondence: Rebecca Strawbridge, Centre for Affective Disorders, Department of Psychological Medicine, Institute of Psychiatry, Psychology and Neuroscience, King's College London, London SE5 8AZ, UK. Email: Becci.strawbridge@kcl.ac.uk

First received 13 Feb 2015, final revision 28 May 2015, accepted 28 May 2015

\section{References}

1 Aslam RW, Bhui K. Parity of Esteem as a guiding principle for commissioning service provision and laws related to health care: what do we know. Ethn Health 2012; 17: 13-5.

2 Millard C, Wessely S. Parity of esteem between mental and physical health BMJ 2014; 349: g6821.

3 Prince M, Patel V, Saxena S, Maj M, Maselko J, Phillips MR, et al. No health without mental health. Lancet 2007; 370: 859-77.

4 World Health Organization. Global Health Estimates Summary Tables. WHO, 2011 (http://www.who.int/healthinfo/global_burden_disease/ estimates_regional/en/index1.html).

5 Patel V, Sumathipala A. International representation in psychiatric literature: survey of six leading journals. Br J Psychiatry 2001; 178: 406-9.

6 Byrne P. Stigma of mental illness: changing minds, changing behaviour Br J Psychiatry 1999; 174: 1-2.

7 Lyons Z. Attitudes of medical students toward psychiatry and psychiatry as a career: a systematic review. Acad Psychiatry 2013; 37: 150-7.

8 Korszun A, Dharmaindra N, Koravangattu V, Bhui K. Teaching medical students and recruitment to psychiatry: attitudes of psychiatric clinicians, academics and trainees. Psychiatrist 2011; 35: 350-3.

9 Gilbody SM, Song F. Publication bias and the integrity of psychiatry research. Psychol Med 2000; 30: 253-8.
10 Stuart H, Sartorius N, Liinamaa T, Images Study Group. Images of psychiatry and psychiatrists. Acta Psychiatr Scand 2015; 131: 21-8.

11 Christensen H, Batterham PJ, Griffiths KM, Gosling J, Hehir KK. Research priorities in mental health. Aust NZ J Psychiatry 2013; 47: 355-62.

12 Vos $T$, Flaxman AD, Naghavi M, Lozano R, Michaud C, Ezzati M, et al. Years lived with disability (YLDS) for 1160 sequelae of 289 diseases and injuries 1990-2010: a systematic analysis for the Global Burden of Disease Study 2010. Lancet 2013; 380: 2163-96.

13 Garfield E. Fortnightly review: how can impact factors be improved? BMJ 1996; 313: 411-3

14 World Health Organization. The World Health Report 2001 - Mental Health New Understanding, New Hope. WHO, 2001.

15 Patel V, Garrison P, de Jesus Mari J, Minas H, Prince M, Saxena S, et al. The Lancet's series on global mental health: 1 year on. Lancet 372: 1354-7.

16 Link BG, Yang LH, Phelan JC, Collins PY. Measuring mental illness stigma Schizophr Bull 2004; 30: 511-41.

17 Paxton R, Kennedy P, Carpenter J. Research in the real world. Psychiatr Bull 2006; 30: 43-5.

18 Evans-Lacko S, Henderson C, Thornicroft G. Public knowledge, attitudes and behaviour regarding people with mental illness in England 2009-2012. Br J Psychiatry 2013; 202 (suppl 55): s51-7.

19 Kakuma R, Minas H, van Ginneken N, Dal Poz MR, Desiraju K, Morris JE, et al. Human resources for mental health care: current situation and strategies for action. Lancet 2011; 378: 1654-63.

20 Friedrich B, Evans-Lacko S, London J, Rhydderch D, Henderson C, Thornicroft G. Anti-stigma training for medical students: the Education Not Discrimination project. Br J Psychiatry 2013; 202 (suppl 55): s89-94.

21 Luty J, Arokiadass SMR, Easow JM, Anapreddy JR. Preferential publication of editorial board members in medical specialty journals. J Med Ethics 2009; 35: 200-2.

22 Proctor EK. Leverage points for the implementation of evidence-based practice. Brief Treat Crisis Interv 2004; 4: 227.

23 Sackett DL, Rosenberg W, Gray J, Haynes RB, Richardson WS. Evidence based medicine: what it is and what it isn't. BMJ 1996; 312: 71-2.

24 Kohn R, Saxena S, Levav I, Saraceno B. The treatment gap in mental health care. Bull WHO 2004; 82: 858-66. 